\title{
Ueber die Constitution des Aldehyds und des Elaylchlorürs ;
}

von Dr. Anlon Geuther.

Wie bekannt hat man früher die Aldehyde den Alkoholen gleich constituirt betrachtet, und wenn man den Alkohol ansah als $\mathrm{C}^{4} \mathrm{H}^{4}$, $\mathrm{HO}+\mathrm{HO}$, hat man sie als $\mathrm{C}^{4} \mathrm{H}^{2}$, $\mathrm{HO}+\mathrm{HO}$ zusammengesetzt annehmen müssen. Später zählle die Gerhard'sche Schule die Aldehyde zu dem Typus $\begin{gathered}\mathrm{H} \\ \mathrm{H}\end{gathered}$, unter Annahme der Ersetzung des einen Wasserstoffs durch das sauerstoffhaltige Radical $\mathrm{C}^{4} \mathrm{H}^{3} \mathrm{O}^{2}$, und damit stand im Einklang, dafs nach Kopp's Untersuchungen der Sauerstoff im Aldehyd ein anderes spec. Volum besilzt, als im Alkohol. Ich kann mich aus Gründen, die ich späler zu entwickeln Gelegenheil nehmen werde, nicht zu dieser Ansicht bekennen, und habe defshalb Untersuchungen über das Verhältnils des Aldebyds zum Elaylchlorür unternommen, zu dem Zwecke, die wahre Constitution des Aldehyds zu ergründen, so wie die des Elaylchlorürs.

Auf den ersten Blick scheinen beide Körper ihren Formeln gemäls ganz nahe verwandt, indem man an eine Substitution des Sauerstoffs im Aldehyd durch Chlor denken kann. Wäre diefs der Fall, so sollte man zu dem Glauben berechtigt sein, das Chlor des Elaylchlorürs liefse sich wieder durch Sauerstoff austauschen, z. B. bei seiner Behandlung mit Silberoxyd. Elaylchlorür wirkt bei der Siedhilze desselben auf Silberoxyd nicht ein; wurde dasselbe aber im verschlossenen Rohr mit 2 Aeq. AgO bis $120^{\circ}$ erhitzl, so fand eine theilweise Zersetzung statt. Es liefs sich die Bildung von Chlorsilber erkennen neben noch unzerselztem Silberoxyd. Die Flüssigkeit in der Röhre sclbst schien unverändert, aber beim Oeffnen des Rohrs zeigte sich eine mächtige Span- 
nung im Innern, denn es genügte ein einziger Feilenstrich, um die Spitze mit hefligem Knall abzuwerfen, wefshalb vorsichtig dabei verfahren werden muls. Die Flüssigkeit war sogleich verschwunden und nur ein laucharliger Geruch wahrnehmbar, gleich dem, welchen die Verbindung $\mathrm{C}^{4} \mathrm{H}^{3} \mathrm{Gl}$ besitzt, die schon bei $-15^{\circ}$ siedet. Aus dem in der Röhre befindlichen Silberoxyd liefs sich durch Ammoniak eine ziemliche Menge Chlorsilber ausziehen. Auf diese Weise war also keine Substitution des Chlors durch Sauerstoff vor sich gegangen.

Ich versuchte, dieselbe durch Kalihydrat zu bewirken, wobei möglicherweise, wenn eine Ersetzung statlfand, die sich bildende Verbindung $\mathrm{C}^{4} \mathrm{H}^{4} \mathrm{O}^{2}$ durch Aufnahme von $2 \mathrm{At}$. HO in Glycolalkohol übergehen konnte. Es wäre diefs letztere nicht unwahrscheinlich gewesen, da aus der Verbindung eines $\mathrm{C}^{4} \mathrm{H}^{4} \mathrm{O}^{2}$ mit 2 At. Essigsäure durch Kalihydrat W urtz Glycolalkohol erhalten halte. Zu diesem Zwecke wurde Elaylchlorür mit überschüssigem Kalihydrat in Röhren eingeschmolzen und im Oelbade erhitzt. Bei einer Temperatur von $130^{\circ}$ bis $140^{\circ}$ explodirten dieselben regelmärsig, delshalb konnte nur eine solche, die bis $125^{\circ}$ ging, angewandt werden. Im Innern des Rohrs fand sich nach mehrstündigem Erhitzen neben überschüssigem Kalihydrat eine ziemliche Menge einer leichtbeweglichen Flüssigkeit. Trotzden die Röhre mit Eis abgekühlt worden war, fand beim Oeffnen heftige Explosion statl und augenblicklich war sämmlliche Flüssigkeit aus der Röhre verschwunden: Es war der nämliche laucharlige Geruch bemerkbar, wie bei dem Versuch mit Silberoxyd. Also auch Kalihydrat hatte nur 1 Atom Chlor wegzunehmen vermoclit, unter Bildung von $\mathrm{C}^{4} \mathrm{H}^{3} \mathrm{Gl}$, Wasser und Chlorkalium. Es scheint defshalb wahrscheinlich, dafs das Elaylchlorür nicht Aldehyd sei, in dem der Sauerstoff durch Chlor erselzt gedachl werden kann. 
Einige Versuche mit Aldehyd, die ich bald werde mittheilen können, führten darauf, den umgekehrlen Weg zu versuchen, zu sehen nämlich, ob Aldehyd sich seiner beiden Atome Sauerstoff berauben und sie durch Chlor ersetzen liefse. Zu dem Ende versuchte ich die Einwirkung von Aldehyd, welches aus krystallisirtem Aldehydammoniak mit Schwefelsäure durch Destillalion im Wasserbade dargestellt worden war, auf Sn $\mathrm{Gl}^{2}$, in der Meinung, dals sich $\mathrm{SnO}^{2}$ und $\mathrm{C}^{4} \mathrm{H}^{4} \mathrm{Gl}^{2}$ bilden möchte. Die Einwirkung beider ist heftig, es entsteht aber SnGl und kein $\mathrm{SnO}^{2}$, wefshalb ich, wenig Hoffnung auf diesen Versuch setzend, mich statt Zinnchlorids des Phosphorsuperchlorids bediente. Beim Zugiefsen des Aldehyds zu dem in einer Retorte befindlichen Phosphorsuperchlorid trat Wärmeentwicklung ein unter Bildung von wenig Salzsäuregas, welches in so geringer Menge auftrat, dals es nur von Spuren Wassers, welches dem Aldehyd beigemischt war, herrühren konnte. Die Relorte wurde durch kalles Wasser kühI gehalten und so lange Aldehyd in kleinen Portionen zugesetzl, als noch ein Ueberschuls von Phosphorsuperchlorid vorhanden war. Beim Erwärmen löste sich alles zu einer homogenen Flüssigkeit auf, welche nun destillirt wurde. In der Retorte trat allmählig Schwärzung und Abscheidung einer kohligen Masse ein. Als das Thermometer bis $100^{\circ}$ gestiegen war, wurde dic Vorlage gewechselt. Das jetzt Uebergehende gab sich beim Vermischen mit Wasser als Phosphoroxychlorid zu erkennen. Das erstere Destillat hingegen mit Wasser rermischt liefs einen öligen Körper fallen, welcher durch Waschen mit Wasser von dem anhängenden Phosphoroxychlorid befreit wurde. Nachdem derselbe mit Chlorcalcium entwässert worden war, wurde or von Neuem destillirt. Fast Alles ging bei dem constanten Siedepunkt von $60^{\circ}$ über (zwischen $59^{\circ}$ und $61^{\circ}$ ), nur ein geringer Theil von höherem Siedepunkt blieb in der Retorte. Das bei $60^{\circ}$ über- 
gegangene Destillat stellt eine wasserhelle Flüssigkeit dar, die einen süfslich ätherischen Geruch und Geschmack besilzt, dem des Chloroforms täuschend ähnlich und ein spec. Gewicht bei $4^{0}, 33=1,189$.

Eine Chlorbestimmung ergah genau den Chlorgehalt des des Elaylcblorürs. Es wurden dazu verwandt: 0,450 Grm. Diese über glühenden reinen Kalk geleitet, wobei nur etwas Geruch nach naphtalinartigen Producten auftrat, lieferten: 1,302 Grm. AgGl $=0,322 \mathrm{Gl}$.

0,539 Substanz mit Kupferoxyd verbrannt, wobei eine Spirale metallischen Kupfers im vordern Theil der Röhre lag, lieferten: 0,2170 Grm. Wasser und 0,4825 Grm. Kohlensäure.

Aus diesen Resultaten berechnet sich folgende Formel:

\begin{tabular}{lcccc} 
& berechnet & & \multicolumn{2}{c}{ gefunden } \\
$\mathrm{C}^{4}$ & 24,24 & & - & 24,44 \\
$\mathrm{H}^{4}$ & 4,04 & & - & 4,47 \\
$\mathrm{G}^{2}$ & $\mathbf{7 1 , 7 1}$ & & $\mathbf{7 1 , 5 5}$ & -
\end{tabular}

Dieser Körper ist also mit dem Elaylchlorür isomer, unterscheidet sich aber von letzterem durch seinen um $22^{\circ}$ niedereren Siedepunkt, sowie durch sein geringeres spec. Gewicht und sein Verhalten zu alkoholischer Kalilösung, wodurch er in der Kälte fast gar nicht zerseizt wird, beim Erwärmen unter Abscheidung von Chlorkalium ebenfalls nur schwierig, denn selbst nach längerem Kochen war der Geruch der Verbindung noch wahrnehmbar. In ein Rohr mit alkoholischer Kalilösung eingeschmolzen und längere Zeit im Wasserbade erhitzt, lieferte er Chlorkalium und eine flüchtige Chlorverbindung, welche bei der Wärme der Hand daraus entwich und mit heller, grüngesäumter Flamme brannte. Er mufs vielleicht mit dem

Oenanthylenchlorür $\mathrm{C}^{14} \mathrm{H}^{14} \mathrm{Gl}^{2}$ and dem

Chlorür des Valeraldehyds $\dot{C^{10}} \dot{H}^{10} \dot{G}^{2}$ 
in eine Reihe gebracht werden, und würde in der Benzoësäurereihe ein entsprechendes Glied im Chlorbenzol $\mathrm{C}^{14} \mathrm{H}^{6} \mathrm{G}^{2}$ haben.

Danach ist es sehr wahrscheinlich, dafs er der wahre Chlorälher des Glycols ist, für welchen Wurtz*) das Elaylchlorür ansieht.

Da nun der Sauerstoff des Aldehyds ein anderes spec. Volum hat, als der der Alkohole, so muls er nothwendig in einer andern Verbindung im Aldehyd enthalten sein, als im Alkohol. In lelzterem aber ist er sehr wahrscheinlich mit Wassersloff verbunden, er mufs defshalb im Aldehyd also sehr wahrscheinlich mit Kohlenstoff verbunden sein, und zwar wäre dann die rationelle Formel des Aldehyds: $\mathrm{C}^{2} \mathrm{H}^{4}, \mathrm{C}^{2} \mathrm{O}^{2}$, d. h. eine Verbindung von Sumpfgas mit Kohlenoxyd.

Demnach wäre die rationelle Formel der oben dargeslellten Chlorverbindung: $\mathrm{C}^{2} \mathrm{H}^{4}, \mathrm{C}^{2} \mathrm{Cl}^{2}$ d. h. sie wäre eine Verbindung von Sumpfgas mit Einfach-Chlorkohlenstoff. Das Elaylchlorür aber müfste betrachtet werden,

$$
\text { als } \mathrm{C}^{4} \mathrm{H}^{2}, \underset{\mathrm{H} G \mathrm{H}}{\mathrm{H}} \quad \text { d. h. }
$$

als der Chloralkohol des Kohlenwasserstoffs $\mathrm{C}^{4} \mathrm{H}^{2}$ (Vinilyl).

$\begin{array}{crr}\text { Gewöhnlicher Alkohol } & \text { Vinylalkohol } & \text { Elaylchlorür } \\ \mathrm{C}^{4} \mathrm{H}^{4}, \mathrm{HO} & \mathrm{C}^{4} \mathrm{H}^{2}, \mathrm{HO} & \mathrm{C}^{4} \mathrm{H}^{2}, \mathrm{HGl} \\ \mathrm{HO} & \mathrm{HO} & \mathrm{HEI}\end{array}$

Das sogenannte Chlorelayl, welches durch alkoholische Kalilösung aus dem Elaylchlorür entsleht, würde als Chlorwasserstoff-Vinilyl $\mathrm{C}^{4} \mathrm{H}^{2}$, $\mathrm{HGl}$ dem Chlorwasserstoff-Elayl $\mathrm{C}^{*} \mathrm{H}^{4}$, HGL (Chloräthyl) analog betrachtet werden müssen.

Man wäre danach nicht zu der Annahme genöthigt, das Elayl sei ein zweibasisches Radical, welches doch gar nicht mit

*) Compt. rend. XLV, 228; diese Annalen CIV, 174. 
der Bildungsweise von Bromäthyl aus demselben, durch einfaches Behandeln mit Bromwasserstoff, wie mil der Darstellung des Alkohols aus demselben, welch beides die schönen Versuche Berthelot's uns gelehrt haben, zu reimen wäre. Wolle man aber seine Zuflucht zu 2 isomeren Elaylen nehmen, die in einander übergehen könnten, würde man nur Hypolhese auf Hypothese bauen, ohne den innern Zusammenhang, der durch die einfachen Beziehungen so schön dargelegt wird, einzusehen.

Aus der obigen Betrachlungsweise des Aldehyds würden sich leicht die zweibasischen Alkohole ableiten lassen. Die Gruppe $\mathrm{C}^{2} \mathrm{H}^{4}, \mathrm{C}^{2} 0^{2}$ würde hier die Stelle des mit einem Atom Wasser verbundenen Elayls (Aether) im gewöhnlichen Alkohol vertreten :

\begin{tabular}{cr} 
gewöhnlicher Alkohol & \multicolumn{1}{c}{ Glycolalkohol } \\
$\left(\mathrm{C}^{4} \mathrm{H}^{4}, \mathrm{HO}\right)$ & $\left(\mathrm{C}^{2} \mathrm{H}^{4}, \mathrm{C}^{2} \mathrm{O}^{2}\right)$ \\
$\mathrm{HO}$ & $\mathrm{HO}, \mathrm{HO}$
\end{tabular}

und die Bibasicilät nothwendig aus der zweibasischen Natur von $\left(\mathrm{C}^{2} \mathrm{H}^{4}, \mathrm{C}^{2} \mathrm{O}^{2}\right)$ folgen:

$$
\begin{aligned}
& \mathrm{C}^{2} \mathrm{H}^{4}=1 \text {-basisch ; } \mathrm{C}^{2} \mathrm{O}^{2}=2 \text {-basisch folglich } \\
& 1+2(-1)=2 \text {. }
\end{aligned}
$$

Es ist bis jetzt nicht gelungen, den Aether des Glycolalkohols, falls man einen andern Körper als das Aldehyd darunter versteht, darzustellen; beim Benzolalkohol

$$
\begin{aligned}
& =\left(\mathrm{C}^{12} \mathrm{H}^{6}, \mathrm{C}^{2} \mathrm{O}^{2}\right) \\
& \text { HO HO }
\end{aligned}
$$

trat an dessen Stelle immer nur Biltermandelöl auf. Delshalb scheint es auch ferner wahrscheinlich, dafs die Aldehyde selbst die Aether der zweibasischen Alkohole sind, so wie sich von selbst aus der zweibasischen Nalur dieser Alkohole ergiebt, dafs die beiden Atome Wasser, welche sie enthalten, dieselbe Rolle spielen, wie das eine Alom im gewöhnlichen Alkohol. 
Diese Betrachtungsweise der Verbindungen wird vielleicht, da sie mit den gegenwärtigen Anschauungen nicht übereinstimnt, elwas auffallend erscheinen, zumal ich sie nicht im nöthigen Zusammenhang hier vortragen kann, sondern nur als Bruchstück späterer Vittheilungen voraus cilirt habe; sie wird aber schon bei näherer Betrachtung wohl etwas ihres, wie mir dünkt, rationellen Charakters verrathen; und flielst aus dem allgemeinen Princip, zu welchem die chemische Wissenschaft nöthigt, alle Verbindungen in binäre aufzulösen, weil dann erst für den forschenden Geist ein Ruhepunlit erreicht ist, von dem aus er weiter in die noch völlig geheimen Verhältnisse der 1somerieen und Allotropieen vordringen kann.

Zum Schlufs dieser Miltheilung knüpfe ich nochmals an die obenerwähnte von Ko p $p^{*}$ ) entdeckte Thatsache an, dafs der Sauerstoff zweierlei spec. Volume besitzt. Betrachtet man alle die sauerstoffhaltigen Verbindungen, für welche genannter Forscher das spec. Volum bestimmt hat, so findet man, dafs es in der That sehr wahrscheinlich ist, der Sauerstoff besilze, je nachdem er mit Wasserstoff in diesen Verbindungen verbunden ist, das spec. Volum 3,9 (aufserhalb des Radicals), oder je nachdem er mil Kohlenstoff verbunden ist das von 6,1 (im Radical).

Im Wasser, den Alkoholen und den einfachen Aethern ist er mit Wasserstoff zu HO verbunden, hat defshalb das spec. Volum 3,9; in den Aldehyden ist er mit Kohlenstoff zu $\mathrm{C}^{2} 0^{2}$ verbunden und hat das spec. Volum 6,1 ; in den Säuren ist er theilweise mit Wasserstoff, theilweise mit Kohlenstoff verbunden. In den fetten Säurers sind 2 At. Sauerstoff mit 2 At. Wasserstoff zu 2 HO und 2 At. Sauerstoff mit 2 At. Kohlenstoff zu $\mathrm{C}^{2} \mathrm{O}^{2}$ verbunden.

*) Diese Annal. XCVI, 165. 
Die Formeln sind:

$$
\begin{aligned}
& { }_{\mathrm{HO}}^{\mathrm{HO}} \mathrm{C}^{2} \mathrm{O}^{2}=\text { Ameisensäure } \\
& \left.\mathrm{C}^{2} \mathrm{H}^{2}, \underset{\mathrm{HO}}{\mathrm{HO}}\right\} \mathrm{C}^{2} \mathrm{O}^{2}=\text { Essigsäure } \\
& \mathrm{C}^{4} \mathrm{H}^{4},{ }_{\mathrm{HO}}^{\mathrm{HO}} / \mathrm{C}^{2} \mathrm{O}^{2}=\text { Propionsäure }
\end{aligned}
$$

Bei der Benzoësäure findet das nämliche Yerhältnifs statt:

$$
\left.\mathrm{C}^{12} \mathrm{H}^{4}, \begin{array}{l}
\mathrm{HO} \\
\mathrm{HO}
\end{array}\right\} \mathrm{C}^{2} \mathrm{O}^{2}
$$

Ebenso bei den Aethern der felten Säuren:

$$
\begin{aligned}
& \mathrm{C}^{4} \mathrm{H}^{4}, \mathrm{HO} / \mathrm{C}^{2} \mathrm{O}^{2}=\text { Ameisensaures Aethyl } \\
& \mathrm{C}^{2} \mathrm{H}^{2}, \mathrm{HO} / \mathrm{C}^{2} \mathrm{O}^{2}=\text { Essigsaures Methyl } \\
& \mathrm{C}^{2} \mathrm{H}^{2}, \mathrm{HO} \mathrm{C}^{2}
\end{aligned}
$$

Bei den wasserfreien felten Säuren (Anhydriden) verhält sich der mit Wasserstoff verbundene Sauerstoff zu dem mit Kohlenstoff verbundenen wie 1: 2 :

$$
\begin{gathered}
\left(\mathrm{C}^{2} \mathrm{H}^{2}, \mathrm{HO}\right) \mathrm{C}^{2} \mathrm{O}^{2} \text { oder } \begin{array}{l}
\mathrm{C}^{2} \mathrm{H}^{2}, \mathrm{HO} / \mathrm{C}^{2} \mathrm{O}^{2} \\
\left.\mathrm{C}^{2} \mathrm{H}^{2}, \mathrm{HO}\right\} \mathrm{C}^{2} \mathrm{O}^{2}
\end{array} \\
\text { oder } \left.\mathrm{C}^{2} \mathrm{H}^{2}, \mathrm{HO} O \mathrm{C}^{2} \mathrm{O}^{2}\right\} \mathrm{C}^{2} \mathrm{O}^{2} \\
\left(\mathrm{C}^{2} \mathrm{H}^{2}, \mathrm{HO}\right) \mathrm{C}^{2} \mathrm{O}^{2}
\end{gathered}
$$

Für alle diese Verbindungen berechnet sich das spec. Volum gleich, mag man dem eben Angeführten beipflichten, oder der Ansicht huldigen, nach welcher der Sauersloff im Radical von dem aufserhalb desselben zu trennen und beiden verschiedenes spec. Vol. zuzuschreiben ist. Anders wird es bei den kohlensauren und oxalsauren Aelhern; hier findet eine Differenz in den Formeln stalt. Für die verschiedenen Formeln berechnet ist das spec. Volum folgendermalsen: 
ber. spec. Vol.

I. $\stackrel{\mathrm{C}^{4} \mathrm{H}^{5}}{\mathrm{C}^{2}} \mathrm{O}^{2}$ $\mathrm{C}^{4} \mathrm{H}^{5} \mathrm{O}^{2}$

$\left.137,8 \begin{array}{l}\mathrm{C}^{4} \mathrm{H}^{4}, \mathrm{HO} \\ \mathrm{C}^{4} \mathrm{H}^{4}, \mathrm{HO}\end{array}\right\} \mathrm{C}^{2} \mathrm{O}^{4} \quad 142,2$

II. $\left.\mathrm{C}^{2} \mathrm{H}^{3} \mathrm{O}^{4}\right) \mathrm{O}^{2}$ $\left.\mathrm{C}^{2} \mathrm{H}^{3}\right)^{\mathrm{O}^{2}}$

$\left.117,0 \begin{array}{ll}\mathrm{C}^{2} \mathrm{H}^{2}, \mathrm{HO} \\ \mathrm{C}^{2} \mathrm{H}^{2}, \mathrm{HO}\end{array}\right\} \mathrm{C}^{4} \mathrm{O}^{6}$

121,4

116,3

III. $\mathrm{C}^{4} \mathrm{O}^{4} / \mathrm{O}^{2}$ $\left.\mathrm{C}^{4} \mathrm{H}^{5}\right)^{\mathrm{O}^{2}}$ ber. sp. Yol. beob. sp. Yol.

Die Differenz im spec. Volum der Verbindungen für die verschieden betrachteten Sauerstoffatome beträgt nur 4,4, sie ist also so gering, dafs sie innerhalb der Fehlargrenzen, welche sich bei Vergleichung beobachteter und berechneter spec. Volumina herausslellen, selbst liegt, kann daher hierbei nicht mafsgebend sein. Bei 1. und II. ist die Berechnung nach den andern Formeln übereinstimmender mit der Beobachtung, dagegen bei lll. ergiebt die Berechnung nach dem oben aufgestellien Salz ein bei weitem besseres Resultat. Mit Hülfe der Sauersloffverbindungen wird sich über diese Differenz der Ansichten wohl wenig entscheiden lassen, vielleicht eher schon mil Hülfe der Schwefelverbindungen, wo die Differenz der spec. Volumina der beiden verschicdenen Atome Schwefel schon, wie K o p p ) vermuthet, $=3$ ist, und von wo aus inan durch Analogie von der Constitution des Schwefelkohlenstoffs auf die der Kohlensäure schliefsen kann.

Gö t t ingen, den 7. Januar 1858.

*) Diese Annalen XCVI, 309. 